Chapman University

Chapman University Digital Commons

Psychology Faculty Articles and Research

Psychology

7-9-2014

\title{
Development of a Short Version of the Modified Yale Preoperative Anxiety Scale
}

\author{
Brooke N. Jenkins \\ Chapman University, bjenkins@chapman.edu \\ Michelle Fortier \\ University of California, Irvine \\ Sherrie H. Kaplan \\ University of California, Irvine \\ Linda C. Mayes \\ Yale University \\ Zeev N. Kain \\ Yale University
}

Follow this and additional works at: https://digitalcommons.chapman.edu/psychology_articles

Part of the Anesthesia and Analgesia Commons, Child Psychology Commons, Health and Medical Administration Commons, Health Psychology Commons, and the Other Psychiatry and Psychology Commons

\section{Recommended Citation}

Jenkins, B. N., Fortier, M. A., Kaplan, S. H., Mayes, L. C., \& Kain, Z. N. (2014). Development of a short version of the Modified Yale Preoperative Anxiety Scale. Anesthesia \& Analgesia, 119(3), 643-650. https://doi.org/10.1213/ANE.0000000000000350

This Article is brought to you for free and open access by the Psychology at Chapman University Digital Commons. It has been accepted for inclusion in Psychology Faculty Articles and Research by an authorized administrator of Chapman University Digital Commons. For more information, please contact laughtin@chapman.edu. 


\section{Development of a Short Version of the Modified Yale Preoperative Anxiety Scale}

Background - The modified Yale Preoperative Anxiety Scale (mYPAS) is the current "criterion standard” for assessing child anxiety during induction of anesthesia and has been used in over 100 studies. This observational instrument covers 5 items and is typically administered at 4 perioperative time points. Application of this complex instrument in busy operating room (OR) settings, however, presents a challenge. This investigation we examined the instrument could be modified and made eaiser to use in OR settings.

Methods - This study used qualitative methods, principal components analyses, Cronbach as, and effect sizes to create the mYPAS-Short Form (mYPAS-SF) and reduce time points of assessment. Data were obtained from multiple patients $\left(\mathrm{N}=3798 ; M_{\mathrm{age}}=5.63\right)$, that have been recruited in previous investigations employing the mYPAS over the past 15 years.

Results - Following qualitative analysis, the 'use of parent' item was eliminated due to content overlap with other items. The reduced item set accounted for $82 \%$ or more of the variance in child anxiety, and produced Cronbach $\alpha$ of at least 0.92 . To reduce the number of time points of assessment, a minimum Cohen $d$ effect size criterion of 0.48 change in mYPAS score across time points was used. This led to eliminating the walk to the OR and entrance to the OR time points.

Conclusions - Reducing the mYPAS to 4 items, creating the mYPAS-SF that can be administered at 2 time points, retained the accuracy of the measure while allowing the instrument to be more easily used in clinical research settings. 


\section{Introduction}

Preoperative anxiety is frequently experienced by children undergoing anesthesia and surgery (1) and is associated with a significant number of adverse outcomes such maladaptive behavioral changes and increased postoperative analgesic requirements (1). It is important, therefore, to be able to accurately assess preoperative anxiety levels in children undergoing surgery. Measuring anxiety in the preoperative settings, however, is challenging in young children. First, there is limited time in which children can be observed during the preoperative phase. Second, the operating room (OR) and holding areas are often hectic settings, which can make administration of an observational anxiety measure a burden for health care providers. Third, young children may not be capable of expressing their levels of anxiety verbally either because they are not yet developmentally capable of such communication or are hindered in doing so due to their anxious state. These dilemmas increase the difficulty in accurately observing and assessing child preoperative distress.

To aid in measuring child anxiety before surgery, the modified Yale Preoperative Anxiety Scale (mYPAS) was developed in 1995 (2) and modified in 1997 (3) (see Appendix A). To date, the mYPAS has been employed in $>100$ studies spanning diverse health fields such as anesthesia, surgery, pediatrics, and dentistry (4-9). This measure uses 5 items, each representing a different domain of child anxiety and is used at 4 points in time during the preoperative phase.

Because of the wide use of the measure, shortening the mYPAS may increase its efficiency in clinical studies and eliminate redundancy within the measure. Several previous studies have used this measure $<4$ time points and with $<5$ items, but these studies did not validate the psychometric properties of their methodology $(5,9-12)$. The goals of this study were 
to assess the validity and reliability of a short version the mYPAS and to determine if the measure can be used at fewer preoperative time points. 


\section{Methods}

Over the past 15 years, our research group has conducted multiple studies using the mYPAS(1,8,9, 13-27). Participants of these studies $\left(\mathrm{N}=3798, M_{\text {age }}=5.63, S D_{\text {age }}=2.63\right)$, were recruited from 2 major children's hospitals in the northeastern and southwestern United States and were undergoing outpatient surgery with general anesthesia. The majority of participants in the sample studied were male (58\%) and Non-Hispanic Caucasians (78\%).

\section{Modified Yale Preoperative Anxiety Scale}

Content. The mYPAS consists of 5 items (activity, vocalizations, emotional expressivity, state of apparent arousal, and use of parent). Each item has Likert-type response options reflecting behaviors. Children's behavior is rated from 1 to 4 or 1 to 6 (depending on the item) with greater numbers indicating the highest severity within that item (see Appendix 1).

Timing of Administration. The mYPAS is typically administered at 4different time points which include preoperative holding, walk to the operating room (OR), entrance to the OR (child enters the OR but has not yet seen the anesthesia mask), and introduction to the anesthesia mask.

Training. The 5 items of the mYPAS are rated at each of the 4 time points by trained raters. During training, raters first read the mYPAS manual describing the purpose, administration, and scoring of the measure. Second, raters in training and previously trained raters score videos as a group and discuss their scoring decisions. Finally, raters in training score videos alone and have their scores compared to previously trained raters’ scores. Any discrepancies between scores are discussed. This procedure is continued until raters in training have Kappa scores of at least .80 for intra and inter-rater reliability. 
Scoring. Ratings produce 4 mYPAS scores (1 for each time point). Each score is calculated by dividing each item rating by the highest possible rating (i.e., 6 for the 'vocalizations' item and 4 for all other items), adding all of the produced values, dividing by 5, and multiplying by 100 . This calculation produces a score ranging from 23.33 to 100 with higher values indicating higher anxiety. The item "use of parent” may not always be rated because it requires the presence of the parent. A large majority of participants in this sample (see Table 1) were not rated on the 'use of parent' item during various time points due to lack of parental presence. When this item is not rated, the score is calculated by dividing each item rating by the highest possible rating, adding all of the produced values, dividing by 4 , and multiplying by 100 . This calculation produces a score ranging from 22.92 to 100 with greater values indicating greater anxiety. The mYPAS measure has strong internal reliability, interrater reliability, and convergent validity (3). 
Table 1. Means, Number of Participants, and Percent of Missing Data for the Modified Yale Preoperative Anxiety Scale Over the Assessment Points with and without the Use of Parent Item

\begin{tabular}{lccc}
\hline Assessment Point & mYPAS $^{a}$ & $n$ & $\%$ \\
& Means $^{b}(S D)$ & & Missing \\
\hline With Use of Parent Item & & & \\
Preoperative Holding & $36.76(17.02)$ & 2874 & 24 \\
Walk to Operating Room & $38.89(19.97)$ & 2419 & 36 \\
Entrance to Operating Room & $45.55(22.78)$ & 1340 & 65 \\
Introduction of Anesthesia Mask & $52.53(27.18)$ & 1315 & 65 \\
Without Use of Parent Item & & & \\
Preoperative Holding & $35.90(17.11)$ & 2899 & 24 \\
$\quad$ Walk to Operating Room & $38.26(19.94)$ & 2634 & 31 \\
$\quad$ Entrance to Operating Room & $42.48(21.97)$ & 3259 & 14 \\
Introduction of Anesthesia Mask & $49.06(26.18)$ & 3178 & 16 \\
\hline
\end{tabular}

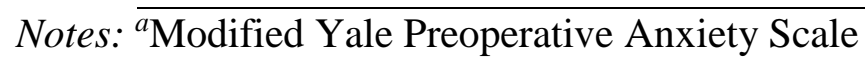

${ }^{b}$ Repeated measures analyses of variance revealed that mYPAS scores at each of the time points were significantly different from one another with the use of parent item $F(2.142,1917.517)=$ 155.368, $\mathrm{p}<.001$, and without the use of parent item $F(2.247,5137.156)=304.574, p<.001$. 


\section{Statistical Analytic Approach}

Eliminating Items. Content analysis of the items was used to determine if any items’ content overlapped with content in the other items. This was accomplished by comparing each response option within an item to all other scale points of the other items. If all the response options in 1 item were represented in response options of other items, the item was eliminated.

After eliminating item(s), the reduced item set was then compared to the original full set within each time point. First, confirmatory principal components analyses (using SPSS version 21; IBM Corp, Armonk, NY) were done to ensure the integrity of the scale was preserved. These analyses examined the variance accounted for in the original item set compared to the reduced item set. If the variance accounted for was within 5\%, the reduced item set was considered to be comparable.

Second, the changes in Cronbach $\alpha$ from the original item set to the reduced set, within each of the time points, were examined. The Cronbach $\alpha$ can range from 0 to 1 , with higher values indicating greater internal consistency. If the Cronbach $\alpha$ for the reduced item set was $>0.90$ (based on previous literature) (28) or higher than the Cronbach $\alpha$ of the original item set, it was considered acceptable.

Eliminating Time Points. The mYPAS produces 4 scores, 1 score for each of the 4 time points. It is possible that these scores are redundant and therefore do not add unique information for research or clinical purposes when assessing child preoperative anxiety. Therefore, it was determined whether the mean mYPAS scores at each of the time points had minimally clinically important (as opposed to statistically significant) differences from one another (29). To establish a minimally clinically important difference (MCID), we used a distribution based approach in which we examined the effect sizes of mYPAS score differences within the literature. Studies 
were identified through the search terms "mYPAS” \& “intervention” in PubMed and Google Scholar, and a recent review of articles on the topic (30). Studies that were selected for use in the table met the following conditions: had a control group receiving no treatment for preoperative anxiety and an intervention group receiving treatment for preoperative anxiety; used the mYPAS as a measure of preoperative anxiety; presented the sample sizes, means, and standard deviations of the groups; provided data on intervention effects; were written in English; and included samples generalizable to our study population (Table 2). Effect sizes of Cohen $d$ among studies comparing children in control conditions to children receiving an intervention were examined to establish a MCID- Cohen $d$ (31) measure of effect size is the difference between the 2 means divided by the pooled standard deviation of both means. Effect sizes of $0.20,0.50$, and 0.80 represent small, medium, and large effects respectively. We determined that the smallest effect size representing the reduction in mYPAS score due to the treatment represent a MCID. Subsequent time points that did not produce this effect, starting from baseline, were eliminated. 
Template for Research Report, Anesthesia \& Analgesia

Copyright (C) 2013 by the International Anesthesia Research Society

Table 2. Effect Sizes Between Control and Intervention Groups ${ }^{\mathrm{a}}$

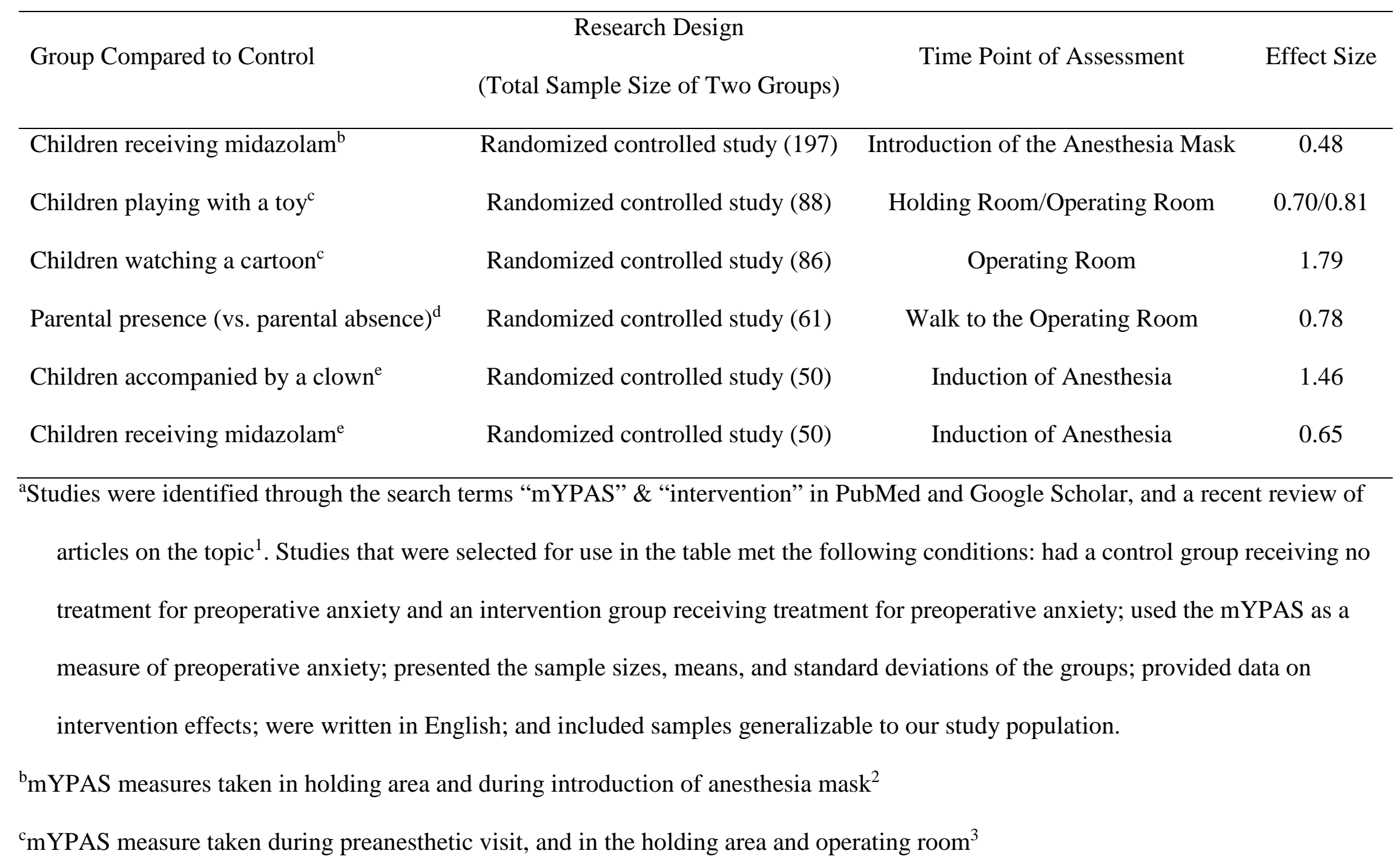


${ }^{\mathrm{d}}$ mYPAS measure taken 90 minutes before surgery, 5 minutes before surgery, walk to the operating room (when children in the parental absence group were separated from their parents), and induction of anesthesia ${ }^{4}$

${ }^{\mathrm{e}} \mathrm{mYPAS}$ measure taken in the holding area and at induction of anesthesia ${ }^{5}$

1. Scully SM: Parental presence during pediatric anesthesia induction. AORN J 2012; 96: 26-33

2. Kain ZN, Caldwell-Andrews AA, Mayes LC, Weinberg ME, Wang SM, MacLaren JE, Blount RL: Family-centered preparation for surgery improves perioperative outcomes in children: a randomized controlled trial. Anesthesiology 2007; 106: 65-74 3. Lee J, Lee J, Lim H, Son JS, Lee JR, Kim DC, Ko S: Cartoon distraction alleviates anxiety in children during induction of anesthesia. Anesth Analg 2012; 115: 1168-73

4. Wright KD, Stewart SH, Finley GA: When are parents helpful? A randomized clinical trial of the efficacy of parental presence for pediatric anesthesia. Can J Anaesth 2010; 57: 751-8

5. Vagnoli L, Caprilli S, Messeri A: Parental presence, clowns or sedative premedication to treat preoperative anxiety in children: what could be the most promising option? Paediatr Anaesth 2010; 20: 937-43 


\section{Results}

\section{Eliminating Items}

Selecting Items to Eliminate. Content analysis was used to eliminate items that were a duplication of other items. The items of “activity”, “vocalizations”, “emotional expressivity”, and "state of apparent arousal" did not have all of their behaviors present in other scale points and as such were not eliminated (Appendix 2). The use of parent item, however, was excluded because all the content within this item was also present in the “activity” item. For example, "may sit close to parent while waiting” and "may push mask away or cling to parent” are behaviors that can be selected within the ‘activity’ item. The item use of parent was also eliminated for practical purposes, because it is not always possible to rate this item because it requires that the parent be present during induction of anesthesia. Because parents are not always present, this item is often not scored (Table 1).

Assessing Reduced Item Set. Confirmatory principal components analyses of all 5 items (full item set) during each of the 4 time points (Appendix 3) were conducted to obtain the percentage of variance accounted for in mYPAS scores. Each analysis produced 1 factor that accounted for at least $80 \%$ of the variance (Table 3). Confirmatory principal components analyses of the reduced item set for each of the time points (see Appendix 3) each produced 1 factor that accounted for at least $82 \%$ of the variance in mYPAS score (Table 3). Each difference between the variances accounted for in mYPAS score during each time point was not $>5 \%$. Therefore, the variance accounted for was considered comparable, indicating that the negative impact of eliminating the item was negligible.

The Cronbach $\alpha$ s of the full item set (all 5 items) during each time point were at least 0.93 (see Table 3). The Cronbach $\alpha$ s for the reduced item set (4 items) during each time point 
were at least 0.92 . All $\alpha$ values were above 0.90 . Therefore, the $\alpha$ values of the reduced item set (now referred to as the mYPAS-Short Form [SF]) were considered to be sufficient, indicating that the internal reliability of the measure was not compromised when removing 1 item.

\section{Eliminating Time Points}

Establishing a Minimally Clinically Important Difference. Table 2 presents the effect sizes of Cohen $d$ among studies comparing children in control conditions to children receiving an intervention. We determined that the smallest effect size of 0.48 , representing the reduction in mYPAS score due to midazolam, is an MCID. This effect size served as our criterion for the elimination of time points.

Comparing Time Points. The means differences between time points of assessment and the pooled standard deviations of mYPAS scores presented in Tables 4 and 5 were used to calculate effect sizes between time points. Although all differences in mYPAS score among time points were statistically significantly different from one another, effect size measures were used to examine the MCID's between the mYPAS scores.

Tables 4 and 5 present the effect sizes between each of the time points for the original mYPAS and the mYPAS-SF, respectively. Because changes in mYPAS score from preoperative holding to walk to the OR and from holding to entrance to the OR produced effect sizes below the set criterion of 0.48 , these 2 time points were eliminated. The change in mYPAS score from preoperative holding to introduction of the anesthesia mask produced effect sizes of 0.53 and 0.48 for the original mYPAS and the mYPAS-SF, respectively. Because these changes were at or greater than the set criterion, this time point was retained. Although the change from walk to the OR to introduction of the anesthesia mask was above the set criteria of 0.48 in Table 4 , the prior elimination of the time point walk to the OR renders that change not useful. 
Table 3. Variance Accounted For by Full Item Set Compared to Reduced Item Set and Cronbach's Alphas of Full Item Set and Reduced Item Set at Each Assessment Point

\begin{tabular}{|c|c|c|c|c|}
\hline \multirow[t]{2}{*}{ Assessment Point } & \multicolumn{2}{|c|}{ Variance Accounted For } & \multicolumn{2}{|c|}{ Cronbach's Alpha } \\
\hline & Full Item Set & Reduced Item Set & Full Item Set & Reduced Item Set \\
\hline Preoperative Holding & $80 \%$ & $82 \%$ & .93 & .92 \\
\hline Walk to Operating Room & $85 \%$ & $86 \%$ & .95 & .94 \\
\hline Entrance to Operating Room & $86 \%$ & $87 \%$ & .95 & .94 \\
\hline Introduction of the & & & & \\
\hline Anesthesia Mask & $90 \%$ & $91 \%$ & .96 & .95 \\
\hline
\end{tabular}


Table 4. Effect Sizes between Assessment Points of Administration of the Modified Yale Preoperative Anxiety Scale (Five Items)

\begin{tabular}{|c|c|c|c|c|c|}
\hline & & Pooled & & & \\
\hline & Mean & & & Effect & $95 \%$ CI on \\
\hline Assessment Points Compared ${ }^{\mathrm{a}}$ & Difference ${ }^{b}$ & Standard & p-value & Size & Effect Si \\
\hline
\end{tabular}

\begin{tabular}{lcccccc}
\hline Holding vs. Walk to OR & 2.52 & 20.29 & $<.001$ & 0.12 & $(0.08,0.15)$ \\
Holding vs. Entrance to OR & 7.72 & 22.84 & $<.001$ & 0.34 & $(0.29,0.40)$
\end{tabular}

Holding vs. Introduction to

$14.83 \quad 28.18 \quad<.001 \quad 0.53 \quad(0.47,0.63)$

Anesthesia Mask

Walk to OR vs. Entrance to OR $5.56 \quad 15.15 \quad<.001 \quad 0.37 \quad(0.31,0.43)$

Walk to OR vs. Introduction of

$12.96 \quad 23.04 \quad<.001 \quad 0.56 \quad(0.49,0.63)$

Anesthesia Mask

Entrance to OR vs. Introduction

of Anesthesia Mask

$\begin{array}{lllll}7.43 & 16.86 & <.001 & 0.44 & (0.38,0.50)\end{array}$

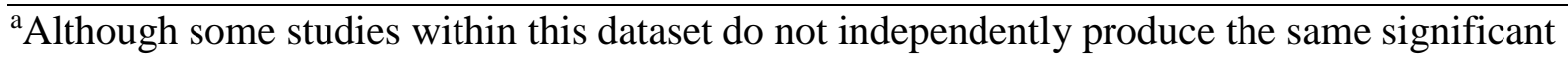
results almost all of the studies produce the same pattern of results.

${ }^{\mathrm{b}}$ Mean differences represent the increase in mYPAS score from the first time point to the second.

${ }^{\mathrm{c}} \mathrm{P}$-values remain significant when Bonferroni correction for familywise error is employed. 
Template for Research Report, Anesthesia \& Analgesia

Copyright (C) 2013 by the International Anesthesia Research Society

Table 5. Effect Sizes between Assessment Points of Administration of the Modified Yale

Preoperative Anxiety Scale Short Form (mYPAS-SF; Four Items)

\begin{tabular}{|c|c|c|c|c|c|}
\hline \multirow[b]{3}{*}{ Assessment Points Compared ${ }^{\mathrm{a}}$} & \multicolumn{3}{|c|}{ Pooled } & & \\
\hline & Mean & & & Effect & $95 \%$ CI on \\
\hline & D.cents & Standard & p-value ${ }^{c}$ & & 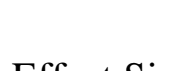 \\
\hline
\end{tabular}

$\begin{array}{lcccccc}\text { Holding vs. Walk to OR } & 2.50 & 20.56 & <.001 & 0.12 & (0.08,0.14) \\ \text { Holding vs. Entrance to OR } & 6.89 & 23.50 & <.001 & 0.29 & (0.26,0.33)\end{array}$

Holding vs. Introduction to

$13.41 \quad 27.97 \quad<.001 \quad 0.48 \quad(0.44,0.52)$

Anesthesia Mask

Walk to OR vs. Entrance to OR

$16.39<.001$

$0.27 \quad(0.23,0.31)$

Walk to OR vs. Introduction of

$10.83 \quad 23.31 \quad<.001 \quad 0.46 \quad(0.42,0.51)$

Anesthesia Mask

Entrance to OR vs. Introduction

of Anesthesia Mask
6.71
$16.56<.001$
0.41
$(0.37,0.44)$

\footnotetext{
${ }^{\mathrm{a} A l t h o u g h}$ some studies within this dataset do not independently produce the same significant results almost all of the studies produce the same pattern of results.

${ }^{\mathrm{b}}$ Mean differences represent the increase in mYPAS score from the first time point to the second.

${ }^{\mathrm{c}} \mathrm{P}$-values remain significant when Bonferroni correction for familywise error is employed.
} 


\section{Discussion}

The purpose of this investigation was to revise the mYPAS, which was developed and validated as an observational measure of children's preoperative anxiety. Because this measure, originally published in Anesthesia and Analgesia,(3) has been in use for >15 years, it was the goal of this study to revisit the mYPAS given the abundance of data that have been collected over the past decade and a half. Accordingly, we have used validated statistical methods to develop the mYPAS-SF (Appendix 4) in an attempt to increase ease of use by removing redundant items. We have also improved the measure by eliminating time points of administration.

Preoperative anxiety is frequently experienced by children undergoing surgery (1) and is associated with a significant number of adverse outcomes such as maladaptive behavioral changes, increased postoperative analgesic requirements, and increased incidence of emergence delirium (2). It is important, therefore, to accurately assess child distress before surgery. However, measuring anxiety in the preoperative setting becomes difficult due to the limited time children can be observed, the hectic settings of the OR and holding areas, and the inability of young children to communicate their anxious state. Because of these challenges, the present study was an opportunity to revise the mYPAS to increase efficiency of its clinical use by addressing barriers to administration.

This investigation resulted in 2 findings. First, the use of parent item was eliminated while retaining the psychometric integrity of the scale. Elimination of this item will shorten the process of training raters to use the mYPAS-SF. Second, using a minimum effect size criterion of change in anxiety across time points of administration resulted in eliminating 2 of the 4 time points. This effectively reduces the time and effort in administration of the mYPAS (or mYPAS- 
SF) in half without any loss of significant clinical information regarding children's preoperative anxiety. Specifically, health care providers or researchers were previously present for the entire preoperative process in order to administer the mYPAS at each of the 4 time points. Eliminating these time points will allow for administration once at the beginning of the preoperative process in the holding area and one final time in the OR when the anesthesia mask is introduced to the child.

In conclusion, we were able to modify the mYPAS to create the mYPAS-SF. This decreased redundancy among items and reduced the time it takes to administer the measure. These revisions increase the clinical applicability of the scale by expanding the use of the measure to health care providers in the busy perioperative clinical settings. 


\section{Appendix 1}

The mYPAS

\section{A. Activity}

1 = Looking around, curious, playing with toys, reading (or other age appropriate behavior); moves around holding area/treatment room to get toys or go to parent; may move toward OR equipment 2 = Not exploring or playing, may look down, may fidget with hands or suck thumb (blanket); may sit close to parent while waiting, or play has a definite manic quality 3 = Moving from toy to parent in unfocused manner, nonactivity derived movements; frenetic/frenzied movement or play; squirming, moving on table, may push mask away or clinging to parent 4 = Actively trying to get away, pushes with feet and arms, may move whole body; in waiting room, running around unfocused, not looking at toys or will not separate from parent, desperate clinging

\section{B. Vocalizations}

1 = Reading (nonvocalizing appropriate to activity), asking questions, making comments, babbling, laughing, readily answers questions but may be generally quiet; child too young to talk in social situations or too engrossed in play to respond 2 = Responding to adults but whispers, "baby talk", only head nodding $3=$ Quiet, no sounds or responses to adults 4 = Whimpering, moaning, groaning, silently crying 5 = Crying or may be screaming "no" 6 = Crying, screaming loudly, sustained (audible through mask) 


\section{Emotional Expressivity}

$1=$ Manifestly happy, smiling, or concentrating on play

$2=$ Neutral, no visible expression on face

3 = Worried (sad) to frightened, sad, worried, or tearful eyes

4 = Distressed, crying, extreme upset, may have wide eyes

D. State of Apparent Arousal

1 = Alert, looks around occasionally, notices watches what anesthesiologist does with him/her (could be relaxed)

2 = Withdrawn child sitting still and quiet, may be sucking on thumb or face turned into adult 3 = Vigilant looking quickly all around, may startle to sounds, eyes wide, body tense 4 = Panicked whimpering, may be crying or pushing others away, turns away

\section{$\underline{\text { E. Use of Parents }}$}

1 = Busy playing, sitting idle, or engaged in age appropriate behavior and doesn't need parent; may interact with parent if parent initiates the interaction 2 = Reaches out to parent (approaches parent and speaks to otherwise silent parent), seeks and accepts comfort, may lean against parent 3 = Looks to parents quietly, apparently watches actions, doesn't seek contact or comfort, accepts it if offered or clings to parent 4 = Keeps parent at distance or may actively withdraw from parent, may push parent away or desperately clinging to parent and will not let parent go Note: Use of parents is only scored when parent is present 
Scoring: Divide each item rating by the highest possible rating (i.e., 6 for the 'vocalizations' item and 4 for all other items), add all of the produced values, divide by 5 (or 4 if $\mathrm{E}$ is not rated), and multiply by 100 . 
Template for Research Report, Anesthesia \& Analgesia

Copyright (C) 2013 by the International Anesthesia Research Society

Appendix 2

List of Item Scale Point Behaviors and Overlapping Content among Other Items

\begin{tabular}{|c|c|c|}
\hline Item & Scale Point Behaviors & Overlapping Content from Other Items \\
\hline \multirow[t]{14}{*}{ Activity } & 1. Looking around, curious, playing with toys, reading (or other age- & "looks around occasionally" - state of arousal \\
\hline & appropriate behavior); moves around holding area/treatment room to & “looking quickly all around” - state of arousal \\
\hline & get toys or go to parent; may move toward OR equipment & “too engrossed in play to respond" - vocalizations \\
\hline & & “reading” - vocalizations \\
\hline & & “engaged in age-appropriate behavior” - use of parent \\
\hline & 2. Not exploring or playing, may look down, may fidget with hands & “May be sucking on thumb” - state of arousal \\
\hline & or suck thumb (blanket); may sit close to parent while waiting & "Reaches out to parent (approaches parent and speaks to otherwise \\
\hline & & silent parent), may lean against parent” - use of parent \\
\hline & 3. Moving from toy to parent in unfocused manner, non-activity & “clings to parent” - use of parent \\
\hline & moving on table, may push mask away or clinging to parent & \\
\hline & 4. Actively trying to get away, pushes with feet and arms, may move & “pushing others away” - state of arousal \\
\hline & whole body; in waiting room, running around unfocused, not looking & “Keeps parent at distance or may actively withdraw from parent, \\
\hline & at toys or will not separate from parent, desperate clinging & may push parent away or desperately clinging to parent and will not \\
\hline & & let parent go” - use of parent \\
\hline
\end{tabular}


Template for Research Report, Anesthesia \& Analgesia

Copyright (C) 2013 by the International Anesthesia Research Society

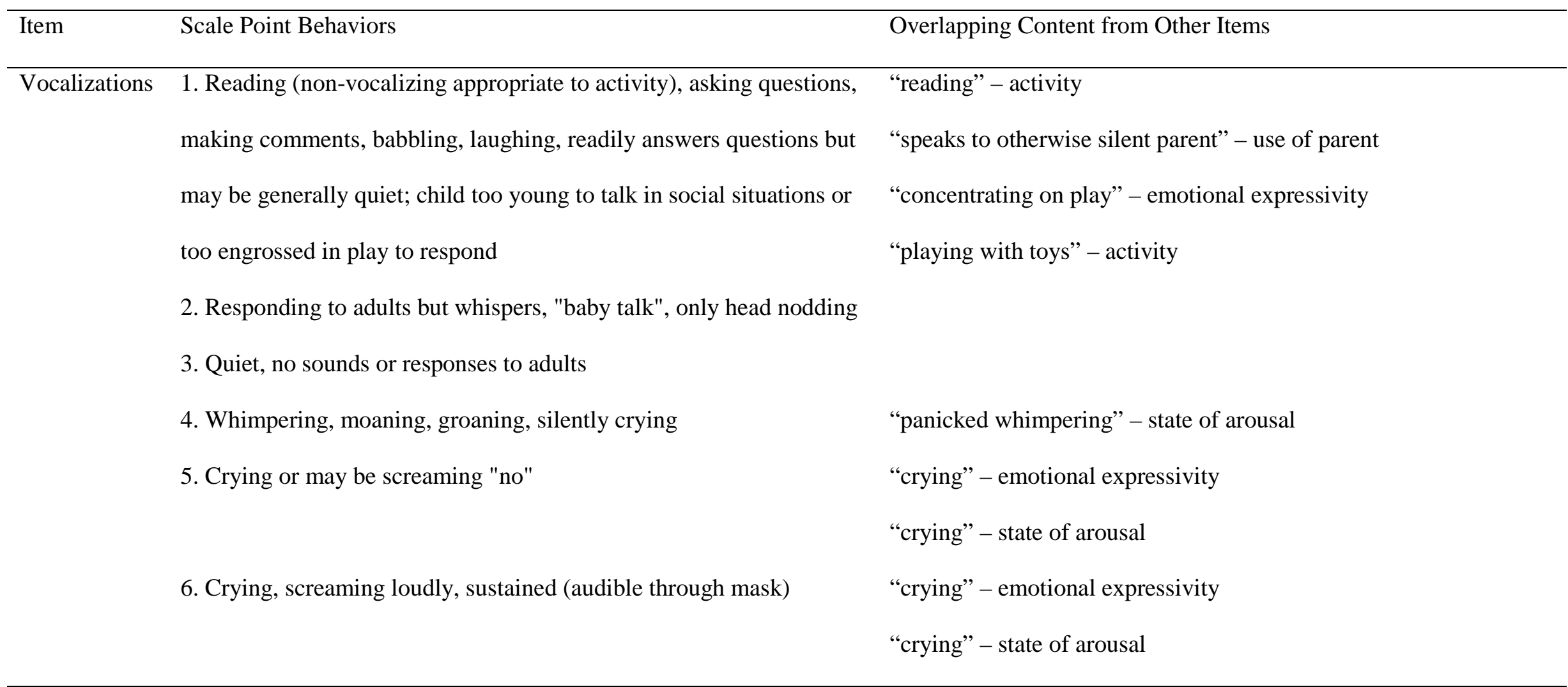


Template for Research Report, Anesthesia \& Analgesia

Copyright (C) 2013 by the International Anesthesia Research Society

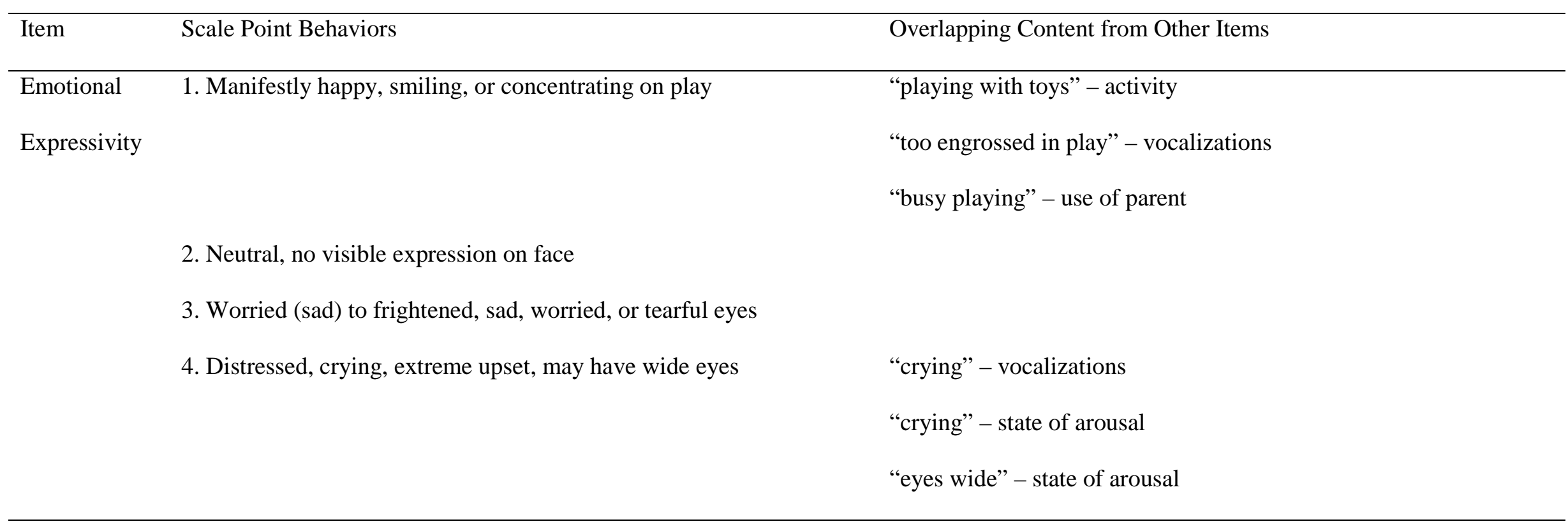


Template for Research Report, Anesthesia \& Analgesia

Copyright (C) 2013 by the International Anesthesia Research Society

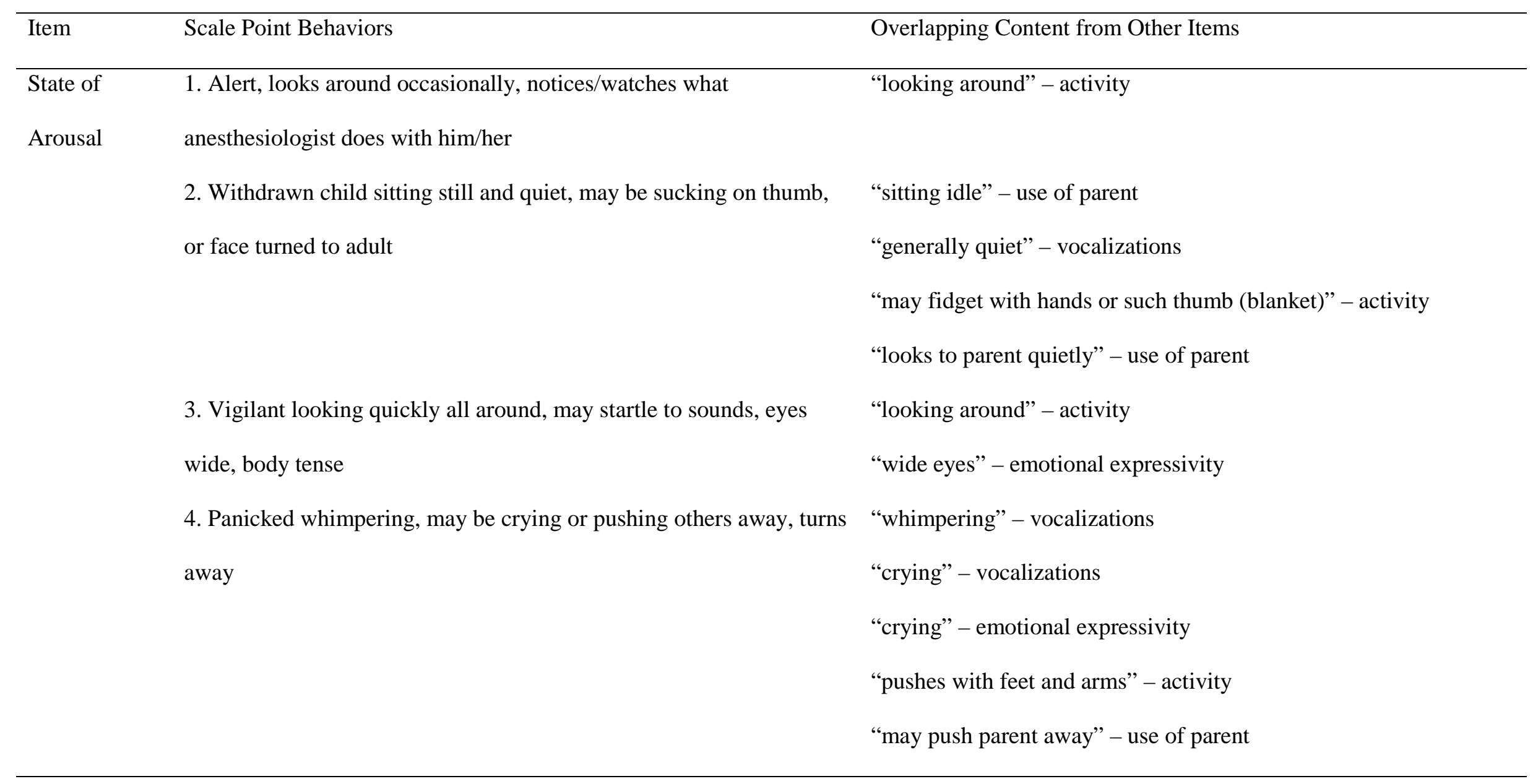


Template for Research Report, Anesthesia \& Analgesia

Copyright (C) 2013 by the International Anesthesia Research Society

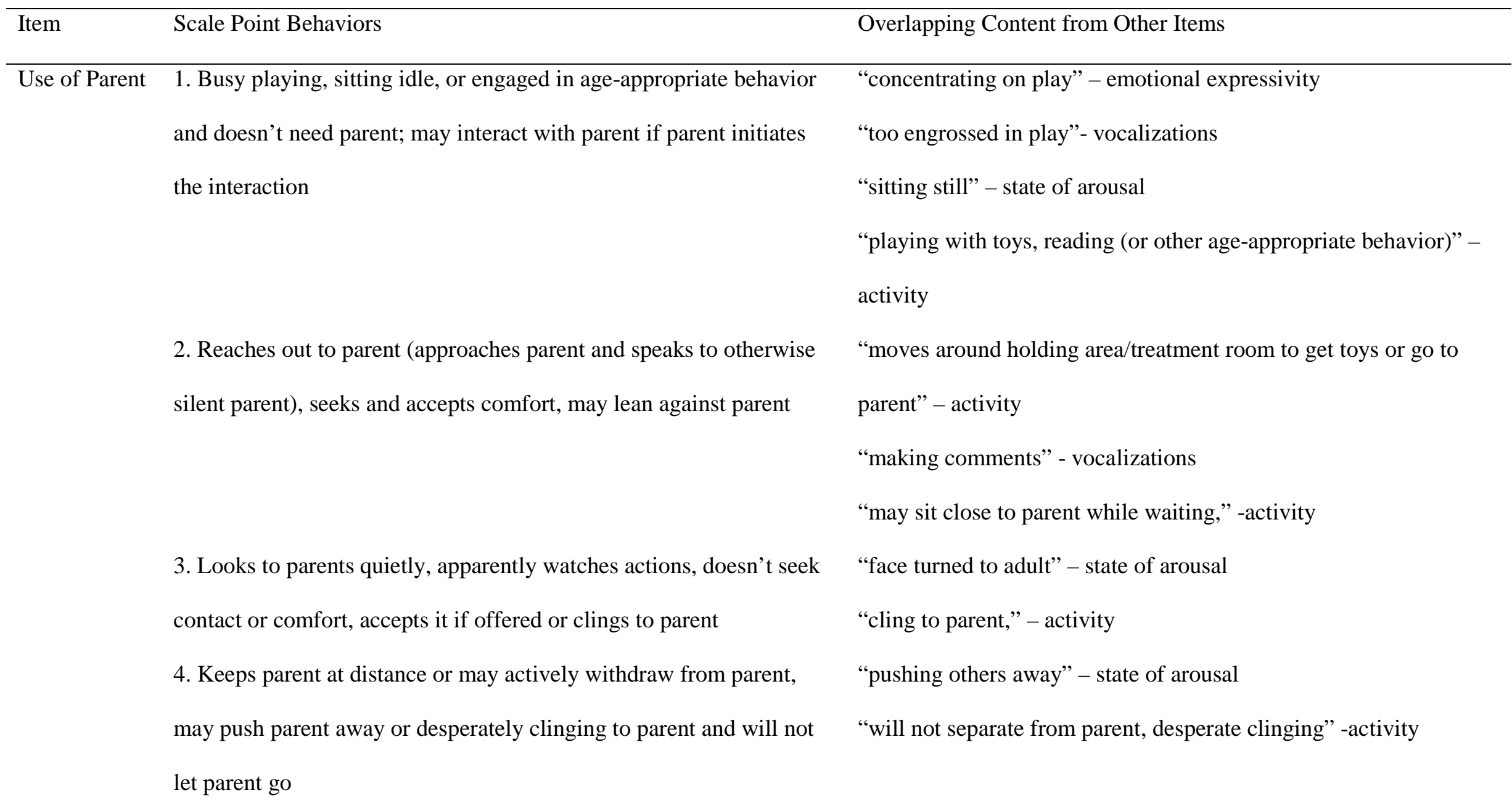


Template for Research Report, Anesthesia \& Analgesia

Copyright (C 2013 by the International Anesthesia Research Society

Appendix 3

Results of the Principal Components Analyses of the mYPAS at the Preoperative Holding Time Point for Full Item Set and Reduced Item Set

\begin{tabular}{lcc}
\hline Item & Full Item Set Factor Loading & Reduced Item Set Factor Loading \\
\hline Activity & .91 & .90 \\
Vocalizations & .86 & .88 \\
Emotional Expressivity & .90 & .91 \\
State of Apparent Arousal & .93 & .93 \\
Use of Parent & .88 & - \\
\hline Eigen Value & 4.00 & 3.28 \\
\% of Variance Explained & 80.0 & 82.0 \\
\hline
\end{tabular}




\section{Appendix 4}

The mYPAS-SF

\section{A. Activity}

1 = Looking around, curious, playing with toys, reading (or other age appropriate behavior); moves around holding area/treatment room to get toys or go to parent; may move toward OR equipment

2 = Not exploring or playing, may look down, may fidget with hands or suck thumb (blanket); may sit close to parent while waiting, or play has a definite manic quality

3 = Moving from toy to parent in unfocused manner, nonactivity derived movements; frenetic/frenzied movement or play; squirming, moving on table, may push mask away or clinging to parent 4 = Actively trying to get away, pushes with feet and arms, may move whole body; in waiting room, running around unfocused, not looking at toys or will not separate from parent, desperate clinging

\section{B. Vocalizations}

1 = Reading (nonvocalizing appropriate to activity), asking questions, making comments, babbling, laughing, readily answers questions but may be generally quiet; child too young to talk in social situations or too engrossed in play to respond

2 = Responding to adults but whispers, "baby talk", only head nodding $3=$ Quiet, no sounds or responses to adults 4 = Whimpering, moaning, groaning, silently crying 
5 = Crying or may be screaming "no"

6 = Crying, screaming loudly, sustained (audible through mask)

C. Emotional Expressivity

1 = Manifestly happy, smiling, or concentrating on play

$2=$ Neutral, no visible expression on face

$3=$ Worried (sad) to frightened, sad, worried, or tearful eyes

4 = Distressed, crying, extreme upset, may have wide eyes

\section{State of Apparent Arousal}

1 = Alert, looks around occasionally, notices watches what anesthesiologist does with him/her (could be relaxed)

2 = Withdrawn child sitting still and quiet, may be sucking on thumb or face turned into adult

3 = Vigilant looking quickly all around, may startle to sounds, eyes wide, body tense

4 = Panicked whimpering, may be crying or pushing others away, turns away

Scoring: Divide each item rating by the highest possible rating (i.e., 6 for the 'vocalizations' item and 4 for all other items), add all of the produced values, divide by four, and multiply by 100 . 
Template for Research Report, Anesthesia \& Analgesia

Copyright (C) 2013 by the International Anesthesia Research Society

\section{References}

1. Kain ZN, Mayes LC, Caldwell-Andrews AA, Karas DE, McClain BC. Preoperative anxiety, postoperative pain, and behavioral recovery in young children undergoing surgery. Pediatrics 2006;118:651-8.

2. Kain ZN, Mayes LC, Cicchetti DV, Caramico LA, Spieker M, Nygren MM, Rimar S. Measurement tool for preoperative anxiety in young children: The Yale Preoperative Anxiety Scale. Child Neuropsychol 1995;1:203-10.

3. Kain ZN, Mayes LC, Cicchetti DV, Bagnall AL, Finley JD, Hofstadter MB. The Yale Preoperative Anxiety Scale: how does it compare with a "gold standard"? Anesthesia \& Analgesia 1997;85:783-8.

4. Cuzzocrea F, Gugliandolo MC, Larcan R, Romeo C, Turiaco N, Dominici T. A psychological preoperative program: effects on anxiety and cooperative behaviors. Paediatr Anaesth 2013;23:139-43.

5. Davidson AJ, Shrivastava PP, Jamsen K, Huang GH, Czarnecki C, Gibson MA, Stewart SA, Stargatt R. Risk factors for anxiety at induction of anesthesia in children: a prospective cohort study. Paediatr Anaesth 2006;16:919-27.

6. Huet A, Lucas-Polomeni MM, Robert JC, Sixou JL, Wodey E. Hypnosis and dental anesthesia in children: a prospective controlled study. Int J Clin Exp Hypn 2011;59:424-40.

7. Weldon BC, Bell M, Craddock T. The effect of caudal analgesia on emergence agitation in children after sevoflurane versus halothane anesthesia. Anesthesia and Analgesia 2004;98:321-6, table of contents.

8. Fortier MA, MacLaren JE, Martin SR, Perret D, Kain ZN. Pediatric pain after ambulatory surgery: Where's the medication? Pediatrics 2009;124.

9. Fortier MA, Del Rosario AM, Martin SR, Kain ZN. Perioperative anxiety in children. Paediatr Anaesth 2010;20:318-22.

10. Rice M, Glasper A, Keeton D, Spargo P. The effect of a preoperative education programme on perioperative anxiety in children: an observational study. Paediatr Anaesth 2008;18:42630.

11. Ferguson GG, Chen C, Yan Y, Royer ME, Campigotto M, Traxel EJ, Coplen DE, Austin PF. The efficacy of oral midazolam for decreasing anxiety in children undergoing voiding cystourethrogram: a randomized, double-blind, placebo controlled study. J Urol 2011;185:2542-6.

12. Kil HK, Kim WO, Han SW, Kwon Y, Lee A, Hong JY. Psychological and behavioral effects of chloral hydrate in day-case pediatric surgery: a randomized, observer-blinded study. J Pediatr Surg 2012;47:1592-9.

13. Fortier MA, Blount RL, Wang SM, Mayes LC, Kain ZN. Analysing a family-centred preoperative intervention programme: a dismantling approach. Br J Anaesth 2011;106:713-8.

14. Chorney JM, Garcia AM, Berlin KS, Bakeman R, Kain ZN. Time-Window Sequential Analysis: An Introduction for Pediatric Psychologists. J Pediatr Psychol 2010.

15. Fortier MA, Del Rosario AM, Rosenbaum A, Kain ZN. Beyond pain: predictors of postoperative maladaptive behavior change in children. Paediatr Anaesth 2010;20:445-53.

16. Chorney JM, Kain ZN. Behavioral analysis of children's response to induction of anesthesia. Anesth Analg 2009;109:1434-40. 
17. Chorney JM, Torrey C, Blount RL, McLaren CE, Chen WP, Kain ZN. Healthcare provider and parent behavior and children's coping and distress at anesthesia induction. Anesthesiology 2009;111:1290-6

18. Kain ZN, Maclaren J, Weinberg M, Huszti H, Anderson C, Mayes L. How many parents should we let into the operating room? Paediatr Anaesth 2009;19:244-9.

19. Kain ZN, MacLaren JE, Hammell C, Novoa C, Fortier MA, Huszti H, Mayes L. Healthcare provider-child-parent communication in the preoperative surgical setting. Pediatric Anesthesia 2009;19:376-84.

20. Kain ZN, MacLaren JE, Herrmann L, Mayes L, Rosenbaum A, Hata J, Lerman J. Preoperative melatonin and its effects on induction and emergence in children undergoing anesthesia and surgery. Anesthesiology 2009;111:44-9.

21. MacLaren JE, Thompson C, Weinberg M, Fortier MA, Morrison DE, Perret D, Kain ZN. Prediction of preoperative anxiety in children: who is most accurate? Anesth Analg 2009;108:1777-82.

22. Thompson C, MacLaren JE, Harris A, Kain Z. Brief report: prediction of children's preoperative anxiety by mothers and fathers. J Pediatr Psychol 2009;34:716-21.

23. MacLaren JE, Kain ZN. Prevalence and predictors of significant sleep disturbances in children undergoing ambulatory tonsillectomy and adenoidectomy. J Pediatr Psychol 2008;33:248-57.

24. Kain ZN, Caldwell-Andrews AA, Mayes LC, Weinberg ME, Wang SM, MacLaren JE, Blount RL. Family-centered preparation for surgery improves perioperative outcomes in children: a randomized controlled trial. Anesthesiology 2007;106:65-74.

25. Kain ZN, MacLaren J, McClain BC, Saadat H, Wang SM, Mayes LC, Anderson GM. Effects of age and emotionality on the effectiveness of midazolam administered preoperatively to children. Anesthesiology 2007; 107:545-52.

26. Caldwell-Andrews AA, Kain ZN. Psychological predictors of postoperative sleep in children undergoing outpatient surgery. Paediatric anaesthesia 2006;16:144-51.

27. Kain ZN, Caldwell-Andrews AA, Mayes LC, Wang SM, Krivutza DM, LoDolce ME. Parental presence during induction of anesthesia: Physiological effects on parents. Anesthesiology 2003;98:58-64.

28. Kelly AM. Does the clinically significant difference in visual analog scale pain scores vary with gender, age, or cause of pain? Acad Emerg Med 1998;5:1086-90.

29. Nunnally JC. Psychometric Theory. 2nd. ed. New York: McGraw-Hill, 1978.

30. Crosby RD, Kolotkin RL, Williams GR. Defining clinically meaningful change in healthrelated quality of life. J Clin Epidemiol 2003;56:395-407.

31. Scully SM. Parental presence during pediatric anesthesia induction. AORN J 2012;96:26-33.

32. Cohen J. Statistical power analysis for the behavioral sciences (2nd ed) Hillsdale, New Jersey: Lawrence Erlbaum Associates, 1988.

33. Schwimmer JB, Burwinkle TM, Varni JW. Health-related quality of life of severely obese children and adolescents. JAMA 2003;289:1813-9.

34. Varni JW, Limbers CA, Bryant WP, Wilson DP. The PedsQL multidimensional fatigue scale in pediatric obesity: feasibility, reliability and validity. International journal of pediatric obesity : IJPO : an official journal of the International Association for the Study of Obesity 2010;5:34-42. 